\title{
Detección de regularidades en taekwondo de alto nivel
}

\section{Regularities detection in high level of taekwondo}

\section{A detecção de regularidades em alto nível taekwondo}

\author{
Cristina González-Prado*, Xavier Iglesias y M. Teresa Anguera ${ }^{2}$ \\ ${ }^{I} I N E F C$-Barcelona Sport Sciences Research Group, Institut Nacional d'Educació Fisica de Catalunya, INEFC - Universitat de Barcelona, \\ ${ }^{2}$ Departamento de Metodología de las Ciencias del Comportamiento. Facultad de Psicologia. Universidad de Barcelona
}

Resumen: El objetivo de este estudio fue analizar las acciones del combate de taekwondo de alto nivel masculino en las 8 categorías de peso oficiales (Minimosca: <54kg, Mosca: 54-58kg, Gallo: 58-62kg, Pluma: 62-67kg, Ligero: $67-72 \mathrm{~kg}$, Superligero: $72-78 \mathrm{~kg}$, Medio: $78-84$, Pesado: $>84 \mathrm{~kg}$ ) en competición. Se diseñó un instrumento de observación ad hoc vinculado a un nuevo instrumento de registro (Drewtina 23), que analizaba las acciones realizadas en los combates. Los criterios de estudio fueron: el tipo de acción ofensiva y acciones defensivas, las acciones técnicas y los asaltos del combate. La observación se realizó en las finales de 6 campeonatos y copas del mundo del 2000 al 2008, con un total de 48 combates de 71 taekwondistas. Para el análisis estadístico descriptivo e inferencial se utilizó el programa PASW Statistics para Windows, y para el análisis de retardos el SDIS-GSEQ. Los resultados revelan que las acciones ofensivas tienen una mayor frecuencia que las defensivas, siendo el contraataque la ofensiva más utilizada. El peso Medio es el que más ofensivas realiza y el Superligero el que más defensivas. La media de acciones por asalto y combate es más alta en el tercer asalto. Más del $95 \%$ de las veces los combates se resuelven al finalizar el tercer asalto. El análisis secuencial de retardos muestra que las acciones ofensivas actúan como favorecedoras de las acciones eficaces y las defensivas como inhibidoras. La ventaja en el marcador actúa como inhibidora de las acciones ofensivas y excitadora de las defensivas en todas las categorías de peso.

Palabras clave: Acciones tácticas, acciones técnicas, asaltos, metodología observacional, análisis secuencial de retardos.

Abstract: The aim of this study was to analyze the actions of male taekwondo combat at high levels in the 8 official weight classes (Mini flyweight: $<54 \mathrm{~kg}$, Flyweight: $54-58 \mathrm{~kg}$, Bantamweight: $58-62 \mathrm{~kg}$, Featherweight: $62-$ $67 \mathrm{~kg}$, Lightweight: $67-72 \mathrm{~kg}$, Super lightweight: $72-78 \mathrm{~kg}$, Middleweight: 78-84, Heavyweight: $>84 \mathrm{~kg}$ ) in competition. An observation instrument was designed ad hoc, binded to a new recording instrument (Drewtina 23), which analyzed the actions taken out in combat. The study criteria were: the kind of offensive and defensive actions, technical actions and combat assaults. The observation was carried out at the finals of 6 championships and world cups from 2000 to 2008 , for a total of 48 combats, involving 71 fighters. For the descrptive and inferential statistical analysis, PASW Statis- tics for Windows was used, and for the lag sequential analysis, SDIS-GSEQ was used. The results show that the offensive actions are more frequent than defensive ones, since counter-attack is the most utilized technique. The Middleweight class is characterized by the highest number of offensive actions, while the Superlightweight class is characterized by the highest number of defensive ones. The mean of actions for assault and combat is higher during the third time. More than $95 \%$ of the times, the fights are resolved at the end of the third time. The lag sequential analysis show that offensive actions act in favor of effective actions, while defensive actions act as inhibitors. The advantage in the scoreboard acts as inhibitor of the offensive actions and excitator of defensive actions in all weight classes.

Key-words: Tactical actions, technical actions, combat assalts, observational methodology, lag sequential analysis.

Resumo: O objetivo do presente estudo foi analisar as açôes de combate de taekwondo masculino de elite relativamente às 8 categorías oficiais de peso (Minimosca: <54kg, Mosca: 54-58kg, Gallo: 58-62kg, Pluma: $62-67 \mathrm{~kg}$, Ligeiro: $67-72 \mathrm{~kg}$, Superligeiro: $72-78 \mathrm{~kg}$, Medio: $78-84$, Pesado: $>84 \mathrm{~kg}$ ) em contexto de competição. Um instrumento de observação ad hoc, vinculado a um instrumento de registo inovador (Drewtina 23), foi utilizado para observar as açôes realizadas nas finais dos campeonatos do mundo de taekwondo entre 2000 e 2008, perfazendo um total de 48 combates de 71 taekwondistas. Realizaram-se as análises estatística descritiva e inferencial através do programa PASW Statistics para Windows, enquanto que o programa SDIS-GSEQ foi utilizado para a análise sequencial de retardos. Os resultados revelam que as açóes ofensivas, em particular a de contra-ataque, apresentam maior frequência do que as defensivas. Os taekwondistas de peso Médio são os que mais açóes ofensivas realizam, sendo a categoria Superligeiro a que mais açôes defensivas envolve. A média de açôes por assalto e por combate é maior no $3^{\circ}$ assalto, sendo que mais de $95 \%$ dos combates terminam neste assalto. A análise sequencial de retardos mostra que as açōes ofensivas favorecem as açōes eficazes, enquanto que as açōes defensivas as inibem. A vantagem no resultado, em todas as categorias de peso, inibe as açôes ofensivas e estimula as defensivas.

Palavras-chave: Tactical actions, technical actions, combat assalts, observational methodology, lag sequential analysis.

\section{Introducción}

Desde la incorporación del taekwondo al programa olímpico en los Juegos de Sydney 2000 se han producido numerosos

\footnotetext{
Dirección para correspondencia [Correspodence address]: Cristina González-Prado. INEFC-Barcelona Sport Sciences Research Group. Institut Nacional d'Educació Física de Catalunya. Avda. de l'Estadi, 1222 (Anella Olímpica de Montjuïc) 08038 Barcelona (Espańa). E-mail: cgonza22@xtec.cat
}

cambios (Albuquerque, Costa, Samulski y Noce, 2008; Cular, Krstulovic y Janovic, 2008) que han influenciado en las características de este deporte. Las modificaciones reglamentarias introducidas han convertido el taekwondo en un arte marcial exigente dónde para ser un competidor de éxito se ha de tener cualidades técnicas, condición física (Marković, Miśigoj-Duraśković y Trninić, 2005), velocidad, fuerza di- 
námica y resistencia (Sadowski, Gierczuk, Miller y Cieśliński, 2012) y un perfil psicológico y motivacional determinado (Albuquerque et al., 2008; Matsushigue, Hartmann y Franchini, 2009). Algunas de estas modificaciones, como la reducción de la duración de los tres asaltos que componen cada combate, han sido especialmente significativas.

En los últimos ciclos olímpicos se ha incrementado su objeto de estudio en el campo científico (González, Iglesias, Mirallas y Esparza, 2011; WTF, 2010 y 2011; Kazemi, Waalen, Morgan y White, 2006). A pesar de que el taekwondo es un arte marcial muy antiguo, existe poca literatura científica sobre su práctica competitiva (Cular et al., 2008; Marković et al., 2005; Sadowski et al., 2012; Pieter y Pieter, 1995; Luk, Hong y Chu, 2001; Iglesias, Gasset, González y Anguera, 2010; Wąsik, 2010; Falcó, Estevan, Menescardi y Ruiz, 2012; Kazemi, Casella y Perri, 2009).

La evolución de este deporte en distintas escuelas, su origen oriental, así como la falta de manuales de referencia, han provocado que no exista un consenso en relación a la terminología utilizada para caracterizar las acciones de combate. Una propuesta de sistematización de los elementos técnicos y tácticos del taekwondo ha sido publicada recientemente (González et al., 2011) y será utilizada en este estudio. En esta clasificación, el ataque y el contraataque se configuran como acciones ofensivas, mientras que las esquivas y los afrontamientos son defensivas. Otros autores (Cular et al., 2008; Kim, Chung y Lee, 1999; Yujin y Zeng, 1999) consideran exclusivamente como acciones ofensivas los ataques, mientras que engloban en las defensivas los contraataques.

La literatura específica de análisis del taekwondo se ha centrado sobre aspectos tácticos (Sadowski et al., 2012; Kazemi, 2009; Santos, Franchini y Lima-Silva, 2011; Estevan, Molina-Garcia, Falcó y Álvarez, 2010), técnicos (González et al., 2011; Pieter y Pieter, 1995; Falcó et al., 2011; Falcó, Estevan y Vieten, 2011), lesiones (Lystad, Pollard y Graham, 2009), factores psicológicos (Bouhlel, Jouini, Gmada, Nefzi, Abdallah y Tabka, 2006; Butios y Tasika, 2007) o de perfiles técnicos y biomecánicos (WTF, 2011; Iglesias et al., 2010; Wąsik, 2009). La metodología que mejor se adapta al estudio de los factores situacionales en los combates de taekwondo, estudiando el comportamiento de los taekwondistas en su entorno natural (Anguera, Blanco-Villaseñor y Losada, 2001), es la metodología observacional, que ya se ha utilizado en diversas investigaciones relacionadas con la actividad física y el deporte (Anguera et al., 2011; Camerino, Chaverri, Anguera y Jonsson, 2012; Gutiérrez-Santiago, Prieto, Camerino y Anguera, 2011; Hong y Tong, 2000; Miarka, Gonçalves, Ferreira, Boscolo, Calmet y Franchini, 2012; Sautu, Garay y Hernández-Mendo, 2009).

El objetivo de este estudio ha sido determinar la tipología de las acciones, su distribución en los asaltos, las técnicas realizadas y los análisis secuenciales derivados de la detección de patrones de conducta, en competición de taekwondo de alto nivel masculino en sus 8 categorías de pesos.

\section{Método}

\section{Diseño}

El diseño del estudio observacional fue N/P/M (nomotético / puntual / multidimensional): nomotético, por analizar la respuesta de los distintos deportistas en función de sus categorías de peso; puntual, pues a pesar de valorarse dos ciclos olímpicos, se analizaron todos los combates de forma transversal, sin importar su evolución en el tiempo; y multidimensional, al incorporar en el estudio los distintos criterios descritos en el instrumento observacional (Anguera, BlancoVillaseñor, Hernández-Mendo y Losada, 2011).

\section{Participantes}

Se analizaron 71 taekwondistas en 96 intervenciones (48 combates) correspondientes a las finales masculinas de 6 competiciones de máximo nivel (4 campeonatos del mundo y 2 copas del mundo) de los dos primeros ciclos olímpicos (2000 al 2008) del taekwondo (WTF, 2010). Se analizaron 8 combates por competición, la final de cada una de las 8 categorías de peso oficiales: Minimosca $(<54 \mathrm{~kg})$, Mosca (54-58 $\mathrm{kg}$ ), Gallo (58-62 kg), Pluma (62-67 kg), Ligero (67-72 kg), Superligero $(72-78 \mathrm{~kg})$, Medio $(78-84 \mathrm{~kg})$ y Pesado $(>84 \mathrm{~kg})$. Existen varios estudios sobre taekwondistas participantes en los Juegos Olímpicos (Kazemi et al., 2006; Cular et al., 2008) pero como criterio de inclusión hemos descartado la valoración de los combates en la competición olímpica debido a que las categorías en estas competiciones olímpicas tienen una agrupación de pesos diferente a la que se realiza en campeonatos regionales, nacionales e internacionales (WTF, 2010).

Todas las imágenes fueron obtenidas de videos oficiales de distribución pública y su tratamiento siguió los criterios éticos descritos en la declaración de Helsinki (Harriss y Atkinson, 2011).

\section{Instrumentos}

Instrumento observacional

Se sistematizaron y codificaron de forma objetiva y específica las acciones y conductas generadas por los taekwondistas espontáneamente en las competiciones (Anguera et al., 2011). Los criterios analizados siguen la propuesta de sistematización de González et al. (2011), y fueron: tipo de acción: ofensiva, defensiva, fintas y penalizaciones; tipo de acción ofensiva: ataque y contraataque (según su relación temporal con la acción del rival: anticipación, simultáneo y posterior); accio- 
nes técnicas de ataque o contraataque: bandal, miro, tuit, dollio, neryo y otras (bakat, kwon, mom dollio f., mom dollio n., nako y yop); y los asaltos 1, 2, 3 y 4 (muerte súbita). Se ha diseñado un instrumento de observación que combina formato de campo y sistema de categorías (Anguera, Magnusson, y Jonsson, 2007), con datos tipo II (concurrentes y eventobase), valorando el orden de los eventos sin tener presente su duración (Bakeman, 1978), y con categorías mutuamente excluyentes intranivel y concurrentes internivel.

\section{Instrumento de registro}

Las imágenes de los Campeonatos y Copas del Mundo se obtuvieron de vídeos oficiales de los campeonatos. El criterio de inclusión fue que la totalidad de los combates reunieran las condiciones de observabilidad, es decir, que todos los combates estuvieran totalmente grabados y la calidad de las imágenes permitiera los registros de todas las acciones de combate. Las imágenes fueron codificadas y registradas con el software Drewtina 23 (González, 2011) creado ad-hoc para el análisis observacional de combates de taekwondo, basado en Microsoft $^{\oplus}$ Office Access ${ }^{\oplus}$ 2007. Este programa presentaba características de registro similares a otros aplicativos de mayores prestaciones (Hernández Mendo, López-López, Castellano, Morales, y Pastrana, 2012), pero con la ventaja de haber sido diseñado de forma exclusiva para el instrumento de observación creado para este estudio.

\section{Procedimiento}

Se analizaron 4960 acciones de combate mediante una observación sistemática, abierta y no participante (Borrie, Jonsson y Magnusson, 2002). Cada uno de los registros se correspondía a la ejecución o ejecuciones técnicas consecutivas de uno de los competidores. Las acciones de cada deportista se representaban en registros independientes, a pesar que la observación se realizó para ambos contrincantes a la vez. Una de las limitaciones de este estudio viene determinada por los cambios sufridos en las reglas de competición del combate de taekwondo (WTF, 2010) durante los dos ciclos olímpicos: asaltos de 2 minutos (2001, 2002, 2003) en vez de 3 (2005, 2007), diferentes puntuaciones si la zona de contacto es el casco o el peto o un asalto extra en caso de empate, entre otros cambios. Estos factores han sido controlados mediante una muestra intencional en la que en cada competición se analizaba un combate por categoría, afectando por igual los cambios a todas las categorías de peso.

Todos los valores fueron tratados mediante el programa Microsoft ${ }^{\oplus}$ Office Excel $^{\oplus}$ 2007, recodificando variables para el cálculo de los estadísticos descriptivos, que permitieron realizar la transformación de los registros cualitativos en variables cuantitativas, de frecuencias y duraciones, correspondientes a los 48 combates. Los registros fueron tratados para su posterior análisis secuencial mediante el programa SDISGSEQ para Windows 4.1.3 (Bakeman y Quera, 2007).

\section{Control de la calidad del dato}

La validez de constructo del instrumento se determinó mediante su coherencia con el marco teórico y por un panel de expertos compuesto por seis técnicos especialistas en taekwondo, con gran experiencia en este deporte, y obteniendo un nivel de acuerdo del 99\%. La fiabilidad se determinó mediante la concordancia inter-observadores (fiabilidad externa) e intra-observador (fiabilidad interna), utilizando el coeficiente Kappa (Cohen, 1968), que superó el valor de 0,90 en todos los análisis. El programa SDIS-GSEQ para Windows 4.1.3. (Bakeman y Quera, 2007) fue utilizado en este análisis. Ambos procesos aseguran la calidad del dato (Blanco y Anguera, 2003) del estudio.

\section{Resultados}

La distribución de frecuencias de los 4960 registros en función de la categoría ha sido analizada mediante el test de chicuadrado $\left(\chi^{2}\right)$ que nos reveló la no existencia de diferencias significativas entre las distintas categorías de peso en la tipología de las acciones, las distintas acciones tácticas y las ofensivas. Sí se apreciaron diferencias $(p<0,05)$ en la técnica utilizada y los tiempos de contraataque. El análisis en función del campeonato analizado detectó diferencias significativas en la distribución de frecuencias en todas las variables del estudio $(p<0,05)$ exceptuando el tiempo de contraataque.

Se transformaron los registros cualitativos en datos cuantitativos, confeccionando una nueva matriz de 48 registros, uno por combate analizado. El análisis descriptivo muestra las distintas categorías del estudio a través de su frecuencia, media y desviación estándar $( \pm \mathrm{DE})$. La distribución normal se confirmó a través de la prueba de Shapiro-Wilk. Las diferencias entre las técnicas utilizadas en cada categoría de peso se determinaron mediante el test no paramétrico de KruskalWallis. Un análisis de la varianza (ANOVA) determinó las diferencias entre acciones utilizadas en los asaltos de cada combate, con la corrección de Bonferroni en los valores significativos. El análisis estadístico fue realizado mediante el programa PASW Statistics para Windows (v.18, SPSS Inc., Chicago, IL), que es una versión reducida y libre del SPSS. 
Tabla 1. Frecuencia por combate según la tipología de las acciones en relación a las 8 categorías de peso en las finales de 6 competiciones internacionales de alto nivel. Valores expresados en media \pm desviación estándar (n) y porcentaje (\%) del total de acciones.

\begin{tabular}{|c|c|c|c|c|c|c|c|}
\hline & \multicolumn{3}{|c|}{ Ofensivas } & Defensivas & Fintas & \multicolumn{2}{|c|}{ Penalizaciones } \\
\hline Categoría & Ataque & Contraataque & Ofensivas & & & & \\
\hline Peso & $n=1491$ & $n=1614$ & $\mathrm{n}=3105$ & $\mathrm{n}=1015$ & $\mathrm{n}=674$ & & $n=166$ \\
\hline Minimosca & $34,3 \pm 12,9$ & $31,7 \pm 10,3$ & $66,0 \pm 22,3$ & $22,2 \pm 13,2$ & $\pm 7,2$ & 5,0 & $\pm 2,0$ \\
\hline $\mathrm{n}=659$ & $52,0 \%$ & $48,0 \%$ & $60,1 \%$ & $20,2 \%$ & $15,2 \%$ & & $4,6 \%$ \\
\hline Mosca & $26,8 \pm 10,1$ & $31,2 \pm 11,9$ & $66,0 \pm 22,3$ & $18,8 \pm 9,0$ & $14,0 \pm 7,1$ & 3,5 & $\pm 3,3$ \\
\hline $\mathrm{n}=566$ & $46,3 \%$ & $53,7 \%$ & $61,5 \%$ & $20,0 \%$ & $14,8 \%$ & & $3,7 \%$ \\
\hline Gallo & $32,5 \pm 8,1$ & $36,0 \pm 17,3$ & $68,5 \pm 22,0$ & $19,7 \pm 10,0$ & $15,3 \pm 14,8$ & 3,0 & $\pm 1,8$ \\
\hline $\mathrm{n}=639$ & $47,4 \%$ & $52,6 \%$ & $64,3 \%$ & $18,5 \%$ & $14,4 \%$ & & $2,8 \%$ \\
\hline Pluma & $33,7 \pm 7,1$ & $32,3 \pm 10,3$ & $66,0 \pm 16,7$ & $22,2 \pm 8,1$ & $12,5 \pm 2,2$ & 3,3 & $\pm 2,1$ \\
\hline $\mathrm{n}=624$ & $51,0 \%$ & $49,0 \%$ & $63,5 \%$ & $21,3 \%$ & $12,0 \%$ & & $3,2 \%$ \\
\hline Ligero & $29,7 \pm 10,0$ & $37,3 \pm 10,3$ & $67,0 \pm 19,3$ & $25,2 \pm 2,8$ & $20,2 \pm 7,9$ & 3,8 & $\pm 2,0$ \\
\hline $\mathrm{n}=697$ & $44,3 \%$ & $55,7 \%$ & $57,7 \%$ & $21,7 \%$ & $17,4 \%$ & & $3,3 \%$ \\
\hline Superligero & $31,8 \pm 15,9$ & $33,2 \pm 15,6$ & $65,0 \pm 30,6$ & $22,5 \pm 11,9$ & $12,0 \pm 6,9$ & 2,2 & $\pm 2,3$ \\
\hline $\mathrm{n}=610$ & $49,0 \%$ & $51,0 \%$ & $63,9 \%$ & $22,1 \%$ & $11,8 \%$ & & $2,1 \%$ \\
\hline Medio & $31,8 \pm 12,2$ & $33,7 \pm 17,2$ & $65,5 \pm 28,5$ & $19,3 \pm 5,0$ & $11,0 \pm 6,4$ & 3,0 & $\pm \quad 2,8$ \\
\hline $\mathrm{n}=593$ & $48,6 \%$ & $51,4 \%$ & $66,3 \%$ & $19,6 \%$ & $11,1 \%$ & & $3,0 \%$ \\
\hline Pesado & $27,8 \quad \pm 8,1$ & $33,7 \pm 14,0$ & $61,5 \pm 19,5$ & $19,3 \pm 6,6$ & $10,7 \pm 7,2$ & 3,8 & $\pm 2,6$ \\
\hline $\mathrm{n}=572$ & $45,3 \%$ & $54,7 \%$ & $64,5 \%$ & $20,3 \%$ & $11,2 \%$ & & $4,0 \%$ \\
\hline $\begin{array}{l}\text { Total } \\
\qquad \mathrm{n}=4960\end{array}$ & $\begin{array}{c}31,1 \pm 10,4 \\
48,0 \%\end{array}$ & $\begin{array}{c}33,6 \pm 12,8 \\
52,0 \%\end{array}$ & $\begin{array}{c}64,7 \pm 21,4 \\
62,6 \%\end{array}$ & $\begin{array}{c}21,1 \quad \pm 8,5 \\
20,5 \%\end{array}$ & $\begin{array}{c}14,0 \quad \pm 8,1 \\
13,6 \%\end{array}$ & 3,5 & $\begin{array}{l} \pm 2,4 \\
3,3 \%\end{array}$ \\
\hline
\end{tabular}

En la tabla 1 se muestra el análisis de frecuencias destacando que del total de 4960 acciones analizadas, el 62,6\% fueron acciones ofensivas, el 20,5\% defensivas, el 13,6\% acciones de finta sin continuidad y el 3,3\% penalizaciones. Del conjunto de acciones ofensivas el $52 \%$ corresponden a contraataques y el $48 \%$ son ataques. En el análisis por combates se ha obser- vado una media de $64,7 \pm 21,4$ acciones ofensivas y $21,1 \pm 8,5$ defensivas en cada combate, lo que representa una acción defensiva por cada tres ofensivas ejecutadas (tabla 1).

Del global de acciones de contraataque, el 59,3\% fueron en simultáneo, el $38,0 \%$ en posterior y el $2,7 \%$ en anticipación. 
Tabla 2. Frecuencia por combate según las acciones por asalto en relación a las 8 categorías de peso en las finales de 6 competiciones internacionales de alto nivel. Valores expresados en media \pm desviación estándar (n) y porcentaje (\%) del total de acciones.

\begin{tabular}{|c|c|c|c|c|c|c|c|c|c|c|}
\hline \multirow{3}{*}{$\begin{array}{l}\text { Categoría Peso } \\
\text { Minimosca }\end{array}$} & \multirow{2}{*}{\multicolumn{2}{|c|}{$\begin{array}{l}\text { Asalto } 1 \\
\mathrm{n}=1480\end{array}$}} & \multirow{2}{*}{\multicolumn{2}{|c|}{$\begin{array}{c}\text { Asalto } 2 \\
\mathrm{n}=1551 \\
\end{array}$}} & \multirow{2}{*}{\multicolumn{2}{|c|}{$\begin{array}{r}\text { Asalto } 3 \\
\mathrm{n}=1922 \\
\end{array}$}} & \multicolumn{2}{|c|}{ Muerte súbita } & \multicolumn{2}{|c|}{ Combate } \\
\hline & & & & & & & & $\mathrm{n}=7$ & \multicolumn{2}{|c|}{$\mathrm{n}=4960$} \\
\hline & 35,5 & $\pm 16,2$ & 31,8 & $\pm 15,0$ & 42,7 & $\pm 17,5$ & & & 110,0 & $\pm \quad 40,1$ \\
\hline $\mathrm{n}=659$ & \multicolumn{2}{|r|}{$32,3 \%$} & \multicolumn{2}{|r|}{$28,9 \%$} & \multicolumn{2}{|c|}{$38,8 \%$} & & & \multicolumn{2}{|c|}{$100 \%$} \\
\hline Mosca & 27,5 & $\pm 11,7$ & 32,5 & $\pm 14,9$ & 34,3 & $\pm 7,7$ & & & 94,3 & $\pm \quad 31,5$ \\
\hline $\mathrm{n}=566$ & \multicolumn{2}{|r|}{$29,2 \%$} & \multicolumn{2}{|r|}{$34,5 \%$} & \multicolumn{2}{|c|}{$36,4 \%$} & & & \multicolumn{2}{|c|}{$100 \%$} \\
\hline Gallo & 32,2 & $\pm 11,4$ & 30,2 & $\pm 11,9$ & 44,2 & $\pm 17,3$ & & & 106,5 & $\pm \quad 37,2$ \\
\hline $\mathrm{n}=639$ & \multicolumn{2}{|r|}{$30,2 \%$} & \multicolumn{2}{|r|}{$28,3 \%$} & \multicolumn{2}{|c|}{$41,5 \%$} & & & \multicolumn{2}{|c|}{$100 \%$} \\
\hline Pluma & 32,2 & $\pm 11,2$ & 32,5 & $\pm 7,3$ & 38,8 & $\pm 10,2$ & 0,5 & $\pm 1,2$ & 104,0 & $\pm \quad 25,2$ \\
\hline $\mathrm{n}=624$ & \multicolumn{2}{|r|}{$30,9 \%$} & \multicolumn{2}{|r|}{$31,3 \%$} & \multicolumn{2}{|c|}{$37,3 \%$} & \multirow{2}{*}{\multicolumn{2}{|c|}{$0,5 \%$}} & \multicolumn{2}{|c|}{$100 \%$} \\
\hline Ligero & 34,7 & $\pm \quad 12,2$ & 39,8 & $\pm \quad 12,0$ & 41,7 & $\pm \quad 7,0$ & & & 116,2 & $\pm \quad 25,3$ \\
\hline $\mathrm{n}=697$ & \multicolumn{2}{|r|}{$29,8 \%$} & \multicolumn{2}{|r|}{$34,3 \%$} & \multicolumn{2}{|c|}{$35,9 \%$} & & & \multicolumn{2}{|c|}{$100 \%$} \\
\hline Superligero & 34,7 & $\pm 15,2$ & 31,5 & $\pm 16,0$ & 34,8 & $\pm 18,1$ & 0,7 & $\pm 1,6$ & 101,7 & $\pm 47,6$ \\
\hline $\mathrm{n}=610$ & \multicolumn{2}{|r|}{$34,1 \%$} & \multicolumn{2}{|r|}{$31,0 \%$} & \multicolumn{2}{|c|}{$34,3 \%$} & \multirow{2}{*}{\multicolumn{2}{|c|}{$0,7 \%$}} & \multicolumn{2}{|c|}{$100 \%$} \\
\hline Medio & 25,8 & $\pm 13,0$ & 31,3 & $\pm 11,4$ & 41,7 & $\pm 9,0$ & & & 98,8 & $\pm 31,0$ \\
\hline $\mathrm{n}=593$ & \multicolumn{2}{|r|}{$26,1 \%$} & & $31,7 \%$ & & $2,2 \%$ & & & & $0 \%$ \\
\hline Pesado & 24,2 & $\pm 9,5$ & 28,8 & $\pm 11,0$ & 42,3 & $\pm 11,6$ & & & 95,3 & $\pm 23,8$ \\
\hline $\mathrm{n}=572$ & & $25,3 \%$ & & $30,2 \%$ & & $4,4 \%$ & & & & $0 \%$ \\
\hline Total & 30,8 & $\pm 12,4$ & 32,3 & $\pm 12,1$ & 40,1 & $\pm 12,5$ & 0,1 & $\pm \quad 0,7$ & 103,4 & $\pm \quad 31,8$ \\
\hline $\mathrm{n}=4960$ & & $29,8 \%$ & & $31,3 \%$ & & $3,8 \%$ & & $0,1 \%$ & & $0 \%$ \\
\hline
\end{tabular}

La tabla 2 muestra que la media de acciones por combate es de $103,4 \pm 31,8$. La categoría de peso con mayor número de acciones por combate es Ligero con $116,2 \pm 25$, mientras que la de menor número de acciones registradas es Minimosca con $94,3 \pm 31,5$ acciones por combate. El tercer asalto es en el que se han registrado más acciones $(38,8 \%)$ y en todas las categorías es el que mayor número de acciones engloba $(40,1 \pm 12,5)$. El segundo asalto ha registrado el $31,3 \%$ de las acciones por combate, mientras que en el primer asalto el 29,8\%. En la muerte súbita sólo hemos observado el $0,1 \%$ de acciones totales de combate, ocurriendo en 2 de los 48 asaltos analizados, uno en categoría Pluma y otro en Superligero (tabla 2). De los 8 pesos el Minimosca es el que más penalizaciones por combate ha recibido $(4,6 \%)$ y el que menos el peso Superligero $(2,1 \%)$.

No existen diferencias significativas en cuanto al número de acciones para el factor categoría de peso, en cambio sí hemos encontrado diferencias significativas en cuanto a las acciones en función del asalto $(p<0,05)$. Las acciones realizadas en el tercer asalto son superiores $(p<0,05)$ a las registradas en el resto de los asaltos, siendo las del asalto de muerte súbita inferiores al resto $(p<0,05)$. No existen diferencias significativas entre las acciones ejecutadas entre el primer y el segundo asalto. 
Tabla 3. Frecuencia de las acciones ofensivas por combate según las técnicas en relación a las 8 categorías de peso en las finales de 6 competiciones internacionales de alto nivel. Valores expresados en media \pm desviación estándar (n) y porcentaje (\%) del total de acciones.

\begin{tabular}{|c|c|c|c|c|c|c|c|c|c|c|c|c|}
\hline \multirow{3}{*}{$\begin{array}{l}\text { Categoría } \\
\text { Peso } \\
\text { Minimosca }\end{array}$} & \multicolumn{2}{|c|}{ Bandal } & \multicolumn{2}{|r|}{ Miro } & \multicolumn{2}{|c|}{ Tuit } & \multicolumn{2}{|r|}{ Dollio } & \multicolumn{2}{|c|}{ Neryo } & \multicolumn{2}{|c|}{ Otras } \\
\hline & \multicolumn{2}{|c|}{$\mathrm{n}=2383$} & \multicolumn{2}{|r|}{$\mathrm{n}=297$} & \multicolumn{2}{|c|}{$\mathrm{n}=157$} & \multicolumn{2}{|r|}{$\mathrm{n}=98$} & \multicolumn{2}{|c|}{$\mathrm{n}=84$} & \multicolumn{2}{|r|}{$\mathrm{n}=86$} \\
\hline & 44,2 & $\pm 12,9$ & 9,3 & $\pm 5,2$ & 3,3 & $\pm 2,4$ & 3,3 & $\pm 2,4$ & 2,5 & $\pm \quad 1,5$ & 3,3 & $\pm 3,2$ \\
\hline$n=396$ & \multicolumn{2}{|c|}{$66,9 \%$} & \multicolumn{2}{|r|}{$14,1 \%$} & \multicolumn{2}{|c|}{$5,1 \%$} & \multicolumn{2}{|r|}{$5,1 \%$} & \multicolumn{2}{|c|}{$3,8 \%$} & \multicolumn{2}{|r|}{$5,1 \%$} \\
\hline Mosca & 44,5 & $\pm 16,5$ & 6,5 & $\pm 6,1$ & 2,8 & $\pm 2,6$ & 1,7 & $\pm 1,4$ & 1,3 & $\pm \quad 1,0$ & 1,2 & $\pm 1,2$ \\
\hline $\mathrm{n}=348$ & \multicolumn{2}{|c|}{$76,7 \%$} & \multicolumn{2}{|r|}{$11,2 \%$} & \multicolumn{2}{|c|}{$4,9 \%$} & \multicolumn{2}{|r|}{$2,9 \%$} & \multicolumn{2}{|c|}{$2,3 \%$} & \multicolumn{2}{|r|}{$2,0 \%$} \\
\hline Gallo & 52,8 & $\pm 22,3$ & 6,0 & $\pm 7,2$ & 6,2 & $\pm 7,3$ & 1,3 & $\pm 1,4$ & 1,7 & $\pm \quad 1,0$ & 0,5 & $\pm 0,8$ \\
\hline $\mathrm{n}=411$ & \multicolumn{2}{|c|}{$77,1 \%$} & \multicolumn{2}{|r|}{$8,8 \%$} & \multicolumn{2}{|c|}{$9,0 \%$} & \multicolumn{2}{|r|}{$1,9 \%$} & \multicolumn{2}{|c|}{$2,4 \%$} & \multicolumn{2}{|r|}{$0,7 \%$} \\
\hline Pluma & 52,5 & $\pm 12,3$ & 5,8 & $\pm \quad 4,2$ & 2,3 & $\pm 1,5$ & 1,0 & $\pm 0,6$ & 2,3 & $\pm \quad 1,8$ & 2,0 & $\pm 1,8$ \\
\hline$n=396$ & \multicolumn{2}{|c|}{$79,5 \%$} & \multicolumn{2}{|r|}{$8,8 \%$} & &, $5 \%$ & & $1,5 \%$ & &, $5 \%$ & & $3,0 \%$ \\
\hline Ligero & 48,7 & $\pm 11,1$ & 6,5 & $\pm 4,5$ & 3,5 & $\pm 3,6$ & 3,5 & $\pm 2,2$ & 2,7 & $\pm \quad 2,4$ & 2,2 & $\pm \quad 2,1$ \\
\hline$n=402$ & & $2,6 \%$ & & $9,7 \%$ & & $2 \%$ & & $5,2 \%$ & & $0 \%$ & & $3,2 \%$ \\
\hline Superligero & 49,5 & $\pm \quad 24,9$ & 7,5 & $\pm \quad 6,9$ & 2,5 & $\pm \quad 3,1$ & 1,5 & $\pm 1,0$ & 1,7 & $\pm \quad 1,6$ & 2,3 & $\pm 2,7$ \\
\hline $\mathrm{n}=390$ & & $5,2 \%$ & & $11,5 \%$ & & $8 \%$ & & $2,3 \%$ & & $6 \%$ & & $3,6 \%$ \\
\hline Medio & 52,3 & $\pm \quad 24,1$ & 4,3 & $\pm 4,1$ & 2,7 & $\pm 3,4$ & 3,2 & $\pm 1,5$ & 0,7 & $\pm \quad 1,0$ & 2,3 & $\pm 2,0$ \\
\hline $\mathrm{n}=393$ & & $9,9 \%$ & & $6,6 \%$ & & $1 \%$ & & $4,8 \%$ & & $0 \%$ & & $3,6 \%$ \\
\hline Pesado & 52,7 & $\pm 20,5$ & 3,5 & $\pm \quad 2,4$ & 2,8 & $\pm 3,0$ & 0,8 & $\pm 0,8$ & 1,2 & $\pm \quad 1,2$ & 0,5 & $\pm 0,5$ \\
\hline $\mathrm{n}=369$ & & $5,6 \%$ & & $5,7 \%$ & & $6 \%$ & & $1,4 \%$ & & $9 \%$ & & $0,8 \%$ \\
\hline Total & 48,0 & $\pm 16,7$ & 6,1 & $\pm 5,3$ & 2,9 & $\pm 3,6$ & 1,9 & $\pm 1,7$ & 1,6 & $\pm \quad 1,4$ & 1,6 & $\pm 2,0$ \\
\hline $\mathrm{n}=3105$ & & $6,7 \%$ & & $9,6 \%$ & & $1 \%$ & & $3,2 \%$ & &, $7 \%$ & & $2,8 \%$ \\
\hline
\end{tabular}

La tabla 3 muestra la distribución de las acciones técnicas realizadas por combate según la categoría. No hay diferencias significativas en cuanto al tipo de técnica realizada para el factor categoría de peso, pero destacamos que el 76,7\% de las acciones técnicas ejecutadas fueron bandal. La categoría de mayor peso (Pesado) fue la que más utilizó el bandal (85,6\% de las acciones ofensivas) y la de menor peso (Mosca) la que menos (66,9\%), aun siendo también la técnica más utilizada. La acción técnica miro chagui fue la siguiente más utilizada por el global de competidores con un $9,6 \%$ de las acciones técnicas, seguida del tuit chagui con un $5,1 \%$, sumando un $8,7 \%$ el conjunto de acciones técnicas restantes.

Atendiendo a que uno de los objetivos específicos del estudio era detectar la existencia de posibles patrones secuenciales en los combates de taekwondo, se ha aplicado la técnica de análisis secuencial de retardos (lag sequential analysis) propuesta por Bakeman (1978) y Sackett (1979) para identificar la existencia de estructuras estables en las conductas que presentan una probabilidad de aparición (probabilidad condicionada u observada) significativamente mayor que la que se esperaría por el efecto del azar (probabilidad incondicionada o esperada). En este caso se ha decidido analizar el conjunto de registros, independientemente del competidor (peto rojo o azul), para valorar la tipología de las secuencias de acciones realizadas. Para determinar la posible existencia de patrones diferenciales en función de las categorías de peso, se han agregado los 6 combates (sesiones) que conforman el total de registros en cada categoría. Se presentan los respectivos valores de residuos ajustados (tabla 4), que para cada una de las ocho categorías de peso permiten detectar la existencia de patrones de conducta excitatorios $(>1,96$, para $p<0,05)$ o inhibitorios $(<-1,96$, para $p<0,05)$. Los datos registrados mediante el programa Drewtina 23 (González, 2011) corresponden a la modalidad de datos secuenciales de multievento, que son de naturaleza concurrente / evento-base (Bakeman, 1978), y se han obtenido directamente del registro efectuado.

En la tabla 4 se presenta el resumen de conductas significativas detectadas al contrastar como conductas criterio la situación en relación al marcador del taekwondista que ejecuta la acción y la eficacia de la acción realizada. Como conductas condicionadas las distintas acciones tácticas, las sanciones (kyongo y kangchum), las técnicas ejecutadas, el carácter de las acciones ofensivas y el tiempo de los contrataques. Los resultados de la prueba binomial, corregida según la propuesta de Allison y Liker (1982), indican que en todas las categorías de peso sólo se han detectado significaciones estadísticas ( $p<$ $0,05)$ en la relación entre acciones ofensivas, defensivas y sanciones, con la eficacia de las acciones y la situación de victoria o derrota del competidor, no así cuando existe un marcador 
igualado entre ambos contendientes. Excepcionalmente, en nificativas con la ejecución de contraataques en la eficacia de el peso Gallo se han detectado en el retardo 0 relaciones sig- la acción.

Tabla 4. Residuos ajustados obtenidos en el análisis secuencial correspondiente a los retardos $-1,0$ y +1 del total de registros en las 8 categorías de peso, con agregación de los combates en cada una de dichas categorías.

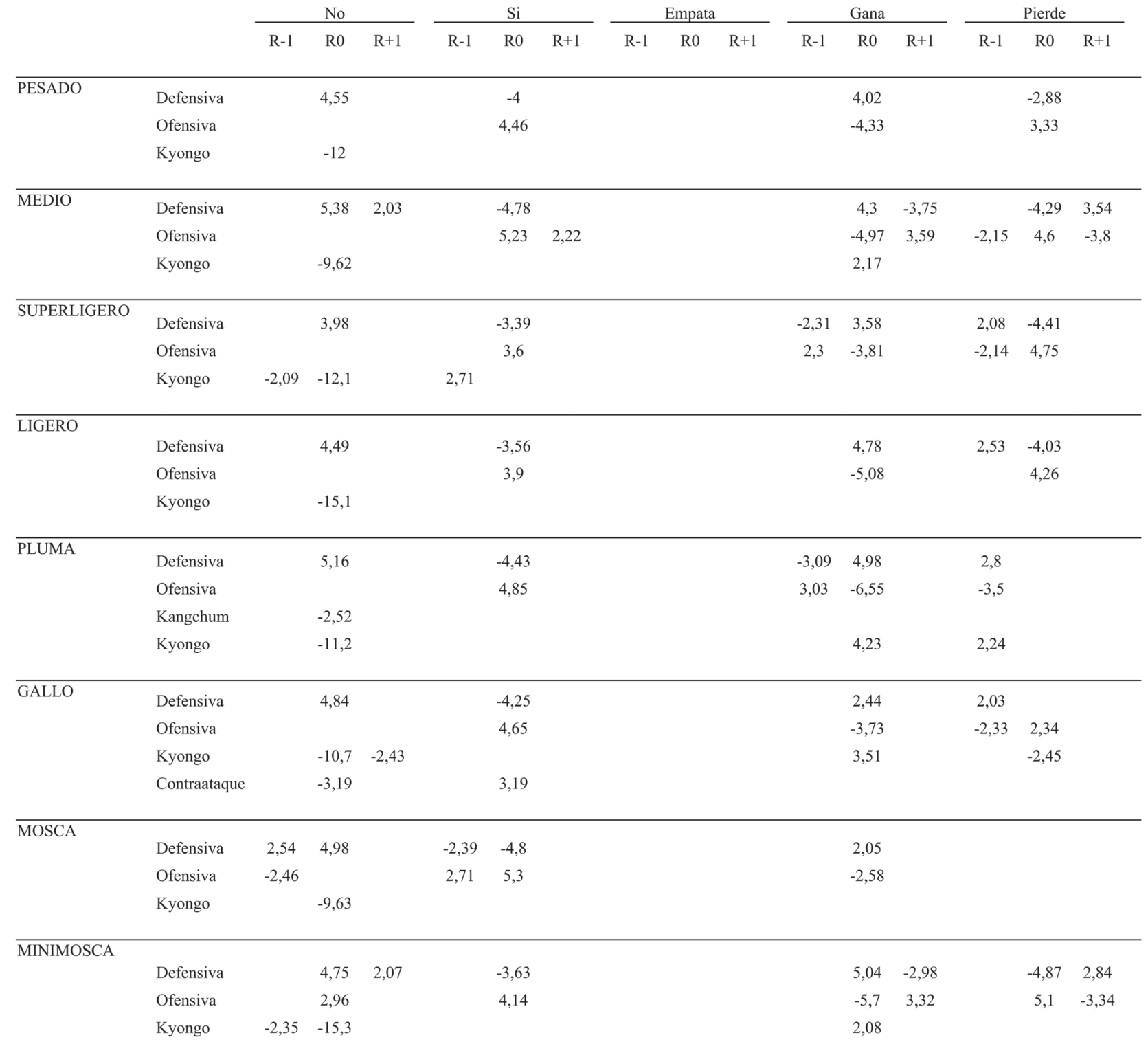

Los residuos ajustados han mostrado, en general, comportamientos similares en las 8 categorías con relaciones significativas en el retardo 0 de todas ellas en la eficacia de las acciones (si/no) y en la situación de ganador de los competidores. Cuando el taekwondista que ejecuta las acciones está en desventaja en el marcador (pierde) no muestra relaciones significativas en retardo 0 en las categorías Pluma y Mosca.

\section{Discusión}

En este trabajo se ha realizado un completo análisis de los mejores taekwondistas del mundo en contexto natural. La valoración de las implicaciones técnicas y tácticas en competición oficial ha sido descrita mediante el recuento de frecuencias y porcentajes del total de registros observados, a través de la estadística descriptiva e inferencial de los datos transfor- 
mados en variables cuantitativas resumen de cada combate $y$, finalmente, del análisis secuencial de las conductas en el desarrollo de los 48 combates de élite analizados.

Las acciones ofensivas con un porcentaje del $62,6 \%$ del total de registros observados y una media de 64,7 acciones ofensivas por combate $( \pm 21,4)$ superan a las defensivas con un $20,5 \%$ del total y valores medios de 21,1 acciones por combate $( \pm 8,5)$. Este porcentaje de acciones ofensivas es superior al 55,8 \% descrito por Luk et al. (2001), aunque el estudio no concreta si los contraataques son considerados como ofensivas o defensivas. La tabla 1 nos muestra como la distribución de las acciones ofensivas está relativamente equilibrada, con una tendencia a existir un mayor número de acciones de contraataque $(52 \%)$ que de ataque (48\%) en el global de ofensivas. Estas cifras muestran como las acciones más ejecutadas en estas seis pruebas de taekwondo del más alto nivel han sido los contraataques, seguidos de los ataques, las acciones defensivas y, finalmente, las fintas sin continuidad. Este porcentaje ligeramente superior de contraataques podría explicarse por el menor riesgo asumido por el deportista, al ejecutarse en función del ataque y al esperar un posible fallo del oponente para obtener cierta ventaja (Kim et al., 1999) y mayor eficacia (González, 2011).

Los resultados descritos son compatibles con la concepción existente del taekwondo como deporte cuyo objetivo es realizar más puntos que el contrario, priorizando las acciones de contraataque a las defensivas, que no puntúan, cuando se producen acciones de ataque.

En el análisis por combates se observa como el tipo de acción ofensiva más utilizado es el contraataque con una media de 33,6 acciones $( \pm 12,8)$ respecto al ataque $(31,1 \pm 10,4)$. Estos porcentajes no se corresponden con todos los estudios consultados en la literatura. En la comparativa de taekwondistas medallistas con no medallistas (Kazemi et al., 2006; Cular et al., 2008) se concluye que los ganadores realizan menos acciones ofensivas y más defensivas -refiriéndose a lo que nosotros denominamos contraataque- en cambio, otras investigaciones (Cular et al., 2008; Santos et al., 2011) coinciden con nuestro trabajo, en que es el ataque el más realizado.

El peso Medio es el que más acciones ofensivas por combate realiza con una media de $65,5 \pm 28,5$ acciones y el peso $\mathrm{Su}$ perligero el que más defensivas con una media de $22,5 \pm 11,9$ acciones. El Minimosca es el que más acciones ofensivas de ataque realiza por combate con una media de 34,3 $\pm 12,9$ y el Ligero el que más acciones ofensivas de contraataque realiza por combate con una media de $37,3 \pm 10,3$ (Tabla 1). Las variaciones en los resultados por categoría coinciden con las observaciones de Craig, Bridge, Jones y Drust (2011) y nos conducen a recomendar una mayor especificidad del entrenamiento del taekwondo en función de las categorías de peso.

Según Yujin y Zeng (1999), el entrenamiento debería centrarse en las técnicas ofensivas y tácticas, y coincidimos con estos autores en que las acciones a entrenar para el combate de taekwondo han de ser las acciones tácticas ofensivas: ataque y contraataque según González et al. (2011), pero también se han de potenciar las acciones tácticas defensivas para evitar que el oponente puntúe.

En cuanto a las amonestaciones observamos que del total de acciones realizadas en todas las categorías de peso, menos de un $5 \%$ son penalizaciones $(3,5 \pm 2,4$ por combate), coincidiendo con los resultados de Kazemi et al. (2006), dónde los taekwondistas ganadores cometen menos faltas que los no ganadores.

De los 8 pesos el Minimosca es el que más penalizaciones por combate ha recibido $(5,0 \pm 2,0)$ y el que menos el peso Superligero $(2,2 \pm 2,3)$. Podría ser lógico pensar que el peso Minimosca, al ser el que más acciones ofensivas de ataque realiza, tiene más opciones de ser amonestado que el resto, en cambio el Superligero es el que más acciones defensivas realiza y esto podría ayudar a realizar menos faltas.

La media de acciones por asalto en los combates se va incrementando del primero $(30,8 \pm 12,4)$ al tercero $(40,1 \pm 2,5)$. Suponemos que a medida que se aproxima el final del combate hay más densidad de acciones porque existe mayor presión temporal (Iglesias et al., 2010) por las necesidades de puntuar ante la reducción del tiempo disponible para hacerlo ante la cercana finalización del combate. Kazemi et al. (2006), en un estudio comparativo realizado con hombres y mujeres medallistas y no medallistas, exponen que tanto los hombres medallistas como los no medallistas obtuvieron el mayor porcentaje de puntos en el primer asalto y las mujeres lo hicieron en el segundo asalto. González (2011) coincide en que el segundo asalto fue más efectivo en un estudio realizado con hombres, y al mismo tiempo coincide con este estudio en que en el asalto en el que más acciones se realizaron fue el tercero, aunque no fue el más efectivo.

De los 48 combates estudiados sólo 2 realizaron la muerte súbita (4,16\%), y esto significa que más del 95\% de las veces los combates finalizan en el tercer asalto. Esta apreciación es de gran interés para planificar los entrenamientos.

La acción técnica más realizada fue el bandal chagui, con $48( \pm 16,7)$ ejecuciones de esta técnica por combate, seguida del miro chagui $(6,1 \pm 5,3)$ y del tuit chagui $(2,9 \pm 3,6)$. Hay diversos estudios sobre el bandal chagui (Pieter y Pieter, 1995; Wąsik, 2010; Estevan et al., 2010; Falcó et al., 2011; Falcó, Alvarez y Castillo, 2009; Estevan, Falcó y Alvarez, 2009) y coincidimos en que es la patada más fácil de realizar por su corta trayectoria, gran velocidad y eficacia, además del poco riesgo que supone realizarla en combate.

El bandal chagui es la patada más ejecutada, con gran diferencia respecto al resto, y coincidimos con otros investigadores (Lee, Chin y Liu, 2005) en que hay un menor tiempo de ejecución de las técnicas lineales frente al resto y que dicha preferencia por las técnicas circulares podría deberse a su alta velocidad y precisión (Pieter y Pieter, 1995). 
Los taekwondistas Pesado, con una media de 52,7 $\pm 0,5$ fueron los que más veces realizaron el bandal chagui, los $\mathrm{Mi}$ nimosca el miro chagui $(9,3 \pm 5,2)$ y los Gallo el tuit chagui $(6,2 \pm 7,3)$. La facilidad de ejecución del bandal chagui permite, a pesos altos con menos movilidad y velocidad que los pesos inferiores, realizar más acciones con un consumo energético inferior.

El Minimosca fue el que menos bandal chagui realizó (Tabla 2), probablemente debido a su mayor agilidad y velocidad (Sadowski, 2012), lo cual les permite realizar otras acciones técnicas de mayor complejidad (mom dollio, neryo, nako) y que les aportan mayor puntuación, como pueden ser las acciones al casco o en salto.

Coincidimos con Kazemi, Perri y Soave (2010) en que las acciones de puńetazo se utilizan fundamentalmente para los intercambios cuerpo a cuerpo, cuando el oponente está en una distancia muy corta. La eficacia de los puńetazos es casi nula (González, 2011). Según las normas de la WTF (2010), para considerar una acción de puñetazo como punto ha de realizarse al peto y con fuerza, y esta última exigencia es muy difícil de valorar por los jueces. En competición, creemos que siempre se ha de responder (contraataque o defensa) con acciones de puńetazo ya que da la sensación de superioridad o al menos de querer seguir luchando y estamos de acuerdo con Cular et al. (2011) en que estos puñetazos se han de entrenar pero no han de ser, evidentemente, el cuerpo principal del entrenamiento.

Mediante el análisis secuencial se pretende poner de manifiesto las relaciones secuenciales entre conductas, identificando las probabilidades de ocurrencia de algunas conductas en función de la ocurrencia previa de otras (Hernández-Mendo y Anguera, 2000). La identificación de conductas de carácter táctico, así como la toma de decisiones, son elementos de gran interés en el rendimiento de este deporte pero poco desarrollados en la literatura, a pesar que en otros deportes de adversario si han sido desarrollados (Martín, González, Cavalcanti, Chirosa y Aguilar, 2013).

La tabla 4 nos muestra los residuos ajustados desde el retardo uno (+1) al menos uno (-1) en que se han encontrado relaciones significativas $(p<0,05)$. De los resultados podemos concluir que son pocas las conductas que generan secuencias significativas.

Si analizamos la categoría de peso Gallo, podemos observar como las acciones tácticas defensivas muestran en retardo cero (0), a nivel de co-ocurrencia, una asociación estadísticamente muy significativa $(4,84)$ con la no eficacia, a la vez que una relación altamente inhibitoria $(-4,25)$ con la eficacia. Estos dos valores se complementan, poniéndose en evidencia que no conviene en ningún caso la estrategia defensiva cuando el objetivo es ganar el punto. La significación excitatoria $(2,44)$ en acciones defensivas cuando el taekwondista va ganando muestra una tendencia a mantener la ventaja en el marcador asumiendo un menor riesgo mediante conductas defensivas. Por el contrario, las acciones tácticas ofensivas han mostrado un residuo ajustado de 4,65, indicando que tiene una relación excitatoria o positiva con la eficacia de las acciones en la consecución de los puntos en competición. Complementariamente a las observaciones realizadas en situación de derrota, cuando el competidor lleva ventaja en el marcador se muestra una relación inhibitoria ante la ejecución de técnicas ofensivas, precisamente por la voluntad de mantener la ventaja sin asumir riesgos de recibir un contraataque durante la acción de ataque. Los elevados valores detectados corroboran el objeto táctico de las acciones implicadas.

En el análisis general de las 8 categorías observamos cómo sólo se han detectado vinculaciones entre las acciones tácticas correspondientes a las defensivas, ofensivas y penalizaciones (Kyongo/Kangchum). Las acciones defensivas generan, en las 8 categorías, una clara relación asociativa estadísticamente significativa $(p<0,05)$ con la no consecución del punto, cuestión previsible porque el objetivo de una acción defensiva no es puntuar sino evitar que el rival lo consiga, por ello, también presenta significación ante la inhibición de conductas que consiguen puntuar. De la misma forma, la aplicación de conductas ofensivas son totalmente favorecedoras de la eficacia $(p<0,05)$ en todas las categorías competitivas. En relación a la situación del marcador también se han detectado regularidades similares en las ocho categorías de competición, siendo las situaciones de ventaja en el marcador excitatorias de la acción defensiva e inhibitoria de las ofensivas en todas ellas. Cuando el deportista está en desventaja en el marcador se produce el efecto contrario en todos los pesos a excepción de Pluma y Mosca.

En conclusión, el tipo de acción ofensiva más utilizado en el taekwondo es el contraataque (52,0\%). El bandal chagui es la acción técnica más utilizada en todas las categorías de peso $(76,7 \%)$. El tercer asalto es en el que más acciones se realizan $(38,7 \%)$ en todas las categorías de peso. El análisis secuencial de retardos no ha mostrado conductas sensibles a la eficacia de las acciones a excepción de las acciones ofensivas, que actúan como favorecedoras y las defensivas como inhibidoras. La ventaja en el marcador actúa como inhibidora de las acciones ofensivas, y excitadora de las defensivas en todas las categorías de peso.

\section{Aplicación práctica}

La sistematización de las observaciones, así como la aplicación de la metodología observacional a deportes como el taekwondo, puede contribuir a una importante mejora del conocimiento de este deporte por parte de los técnicos. La toma de decisiones ante la planificación de las estrategias de entrenamiento debe ser realizada mediante criterios de objetividad, calidad y buscando la optimización del entre- 
namiento de los deportistas. El análisis secuencial contribuirá a detectar regularidades difícilmente observables en el conjunto de situaciones generadas a lo largo de las competiciones. La detección de patrones de conducta permitirá a técnicos y deportistas mejorar las estrategias de análisis de los rivales y preparación de los propios combates. El seguimiento de los propios deportistas y sus rivales podría permitir a los deportistas predecir con menor incertidumbre los comportamientos tácticos de sus rivales. Sería muy interesante la integración de esta metodología en el seguimiento longitudinal de un grupo de deportistas, valorando la incidencia de su incorporación en los procesos de decisión estratégica en los combates.
Agradecimientos: Nuestro reconocimiento a Andrés Fernández por su inestimable aportación en la creación del instrumento de registro Drewtina 23 y a Gabriel Esparza por su ayuda en los procesos de calidad del dato del estudio.

El estudio ha contado con recursos del Institut Nacional d'Educació Física de Catalunya (INEFC Barcelona) y AGAUR (SGR 971 y SGR 1054), y forma parte del proyecto «Observación de la interacción en deporte y actividad física: avances técnicos y metodológicos en registros automatizados cualitativos-cuantitativos», subvencionado por la Secretaría de Estado de Investigación, Desarrollo e Innovación del Ministerio de Economía y Competitividad (DEP2012-32124).

\section{Referencias}

1. Albuquerque, M., Costa, V.T., Samulski, D.M. y Noce, E. (2008). A valiaçâo do perfil motivacional dos atletas de alto rendimento do taekwondo brasileiro. Assessment of the motivational profile of highperformance athletes of Brazilian taekwondo. Revista Iberoamericana de Psicología del Ejercicio y el Deporte, 3, 75-93.

2. Allison, P.D. y Liker, J.K. (1982). Analyzing sequential categorical data on dyadic interaction: A comment on Gottman. Psychological Bulletin, 91(2), 393-403.

3. Anguera, M.T., Blanco-Villaseñor, A. y Losada, J. (2001). Diseños observacionales, cuestión clave en el proceso de la metodología observacional. Metodología de las Ciencias del Comportamiento, 3, 135-160.

4. Anguera, M.T., Blanco-Villaseñor, A., Hernández-Mendo, A. y Losada, J. (2011). Diseńos observacionales: ajuste y aplicación en psicología del deporte. Cuadernos de Psicología del deporte, 11(2), 63-76.

5. Anguera, M.T., Magnusson, M.S. y Jonsson, G.K. (2007). Instrumentos no estándar. Avances en Medición, 5(1), 63-82.

6. Bakeman, R. (1978). Untangling streams of behavior: sequential analysis of observation data. En G. P. Sackett (Ed.), Observing Behavior, Vol. II: Data Collection and Analysis Methods (pp. 63-78). Baltimore: University Park Press.

7. Bakeman, R., y Quera, V. (2007). Software SDIS-GSEQ, versión 4.1.5.

8. Borrie, A., Jonson, G.K. \& Magnusson, M.S. (2002). Temporal pattern analysis and its applicability in sport: An explanation and exemplar data. Journal of Sports Sciences, 20, 845-852.

9. Bouhlel, E., Jouini, A., Gmada, N., Nefzi, A., Abdallah, K.B. y Tabka, Z. (2006). Heart rate and blood lactate responses during taekwondo training and competition. Science Sports, 21, 285-290.

10. Butios, S. y Tasika, N. (2007). Changes in heart rate and blood lactate concentration as intensity parameters during simulated Taekwondo competition. Journal of Sports Medicine and Physical Fitness, 47, 179185.

11. Camerino, O., Chaverri, J., Anguera, M.T. y Jonsson, G.K. (2012). Dynamics of the game in soccer: Detection of T-patterns. European Journal of Sport Science, 12(3), 216-224.

12. Craig, A., Bridge, C.A., Jones, M.A., Drust, B. (2011). The activity profile in international taekwondo competition is modulated by weight category. International Journal of Sports Physiology and Performance, 6, 344-357.

13. Cular, D., Krstulovic, S. y Janovic, M. (2011). The Differences Between Medalists and Non-Medalists at the 2008 Olympic Games Taekwondo Tournament. Human Movement, 12(2), 165-170.

14. Estevan, I., Falco, C., Molina-García, J. y Alvarez, O. (2009). Mechanical Comparison Between Roundhouse Kick To The Chest And To The
Head In Function Of Execution Distance in Taekwondo. 27th International Conference on Biomechanics in Sports, Limerick, Ireland.

15. Estevan, I., Molina-Garcia, J., Falcó, C. y Álvarez, O. (2010). Comparison of efficincy between the roundhouse kick to the chest and to the head according to the execution distance. International Journal of Sport Science, 21, 269-279.

16. Falcó, C., Alvarez, O., Castillo, I., Estevan, I., Martos, J., Mugarra, F. y Iradi, A. (2009). Influence of the distance in a roundhouse kick's execution time and impact force in Taekwondo. Journal of Biomechanics, 42, 242-48.

17. Falcó, C., Estevan, I. y Vieten, M. (2011). Kinematical analysis of five different kicks in taekwondo. Portuguese Journal of Sport Sciences, 11, 219-222.

18. Falcó, C., Estevan, I., Menescardi, C. y Ruiz, L. (2011). The roundhouse kick in function of the two target level. Annual Scientific Congress on Martial Arts and Combat Sports. Viseu, Portugal.

19. Falcó, C., Landeo, R., Menescardi, C., Bermejo, J.L. y Estevan, I. (2012). Match Analysis a University Taekwondo Championship. Advances in Physical Education, 2(1), 28-31.

20. González, C. (2011). Caracterización técnico-táctica de la competición de combate de alto nivel en taekwondo. Efectividad de las acciones tácticas. Tesis Doctoral. Institut Nacional d'Educació Física de Catalunya. Universitat de Barcelona: Barcelona.

21. González, C., Iglesias, X., Mirallas, J.A. y Esparza, G. (2011). Sistematización de la acción táctica en el taekwondo de alta competición. Revista Apuntes Educación Física y Deportes, 103, 56-67.

22. Gutiérrez-Santiago, A., Prieto, I., Camerino, O. y Anguera, M.T. (2011). The temporal structure of judo bouts in visually impaired men and women. Journal of Sports Science, 29(13), 1443-1451

23. Harriss, D., y Atkinson, G. (2011). Update-ethical standards in sport and exercise science research. International Journal of Sports Medicine, 32(11), 819-821.

24. Hernández-Mendo, A., y Anguera, M.T. (2000). Estructura conductual en deportes sociomotores: Hockey sobre patines. Lecturas: Educación Física y Deportes: Revista Digital. Recuperado el 22/2/2001 de: http://www.efdeportes.com/efd21a/hockey.htm.

25. Hernández Mendo, A., López-López, J.A., Castellano, J., Morales, V., y Pastrana, J.L. (2012). HOISAN 1.2: Programa informático para uso en Metodología Observacional. Cuadernos de Psicología del Deporte, 12(1), 55-78

26. Hong, Y., Tong, Y.M. (2000). The playing pattern of the world's top single badminton players in competition a notation analysis. Journal of Human Movement Studies, 38, 185-200. 
27. Iglesias, X., Gasset, A., González, C. y Anguera, M. (2010). Interacción competitiva y presión ambiental en deportes de combate: aplicación de la metodología observacional. Revista Iberoamericana de Psicología del Ejercicio y el Deporte, 5(2), 267-282.

28. Kazemi, M., Casella, C. y Perri, G. (2009). 2004 Olympic Tae Kwon Do Athlete Profile. Journal of the Canadian Chiropractic Association, 53(2), 144-152. Recuperado de http://www.ncbi.nlm.nih.gov/pmc/ articles/PMC2686035/

29. Kazemi, M., Perri, G. y Soave, D. (2010). A profile of 2008 Olympic Taekwondo competitors. Journal of Canadian Chiropractic Association, 54(4), 243-250.

30. Kazemi, M., Waalen, J., Morgan, C. y White, A.R. (2006). A profile of Olympic Taekwondo competitors. Journal of Sports Science \& Medicine, 5, 114-121.

31. Kim, S.H., Chung, K.H. y Lee, K.M. (1999). Taekwondo Kyorugi Olimpic style sparring (p. 224). Wethersfield, U.S.: Turtle Press.

32. Lee, C.L., Chin, Y.F. y Liu, Y. (2005). Comparing the Difference between Front-leg and Back-leg Round-house Kicks Attacking Movement Abilities in Taekwondo. Proceedings of the XXIII International Symposium on Biomechanics in Sports (p. 877-880). Beijing, China.

33. Luk, T.C., Hong, Y. y Chu, D.P.K. (2001). Analysis of strategy used in taekwondo competition. 19th International Symposium on Biomechanics in Sports. University of San Francisco: Blackwell y Sanders.

34. Lystad, R.P., Pollard, H. y Graham, P.L. (2009). Epidemiology of injuries in competition taekwondo: A meta-analysis of observational studies. Journal Sciences Medicine Sport, 12(6), 614-21.

35. Marković, G., Miśigoj-Duraśković, M. y Trninić, S. (2005). Fitness profile of elite Croatian Taekwondo athletes. Collegium Antropologicum, 29, 93-99.

36. Martín, I., González, A., Cavalcanti, L.A., Chirosa, L.J., y Aguilar, J. (2013). Fiabilidad y optimización del programa PROTODEBA v 1.0 para la observación de la Toma de Decisiones en Balonmano. Cuadernos de Psicología del Deporte, 13(1), 63-70.
37. Matsushigue, K., Hartmann, K. y Franchini, E. (2009). Taekwondo: physiological responses and match analysis. Journal of Strength and Conditioning Research, 23(4), 1112-17.

38. Miarka, B., Gonçalves, V.L., Ferreira, U., Boscolo, F., Calmet, M. y Franchini, E. (2012). A comparison of time-motion performance between age groups in judo matches. Journal of Sports Sciences, 30(9), 899905.

39. Pieter, F. y Pieter, W. (1995). Speed and force in selected taekwondo techniques. Biology of Sport, 12, 257-266.

40. Sadowski, J., Gierczuk, D., Miller, J. y Cieśliński, I. (2012). Success factors in elite WTF taekwondo competitors. Achives of Budo, 8, 141146.

41. Sackett, G.P. (1979). The lag sequential analysis of contingency and cyclicity on behavioral interaction research. In J.D. Osofsky (Ed.) Handbook of infant development (pp. 623-649). New York: Wiley.

42. Santos, V.G.F., Franchini, E. y Lima-Silva, A.E. (2011). Relationship between attack and skipping in Taekwondo contests. Journal of Strength and Conditioning Research, 25, 1743-1751.

43. Sautu, L.M., Garay, J.O. y Hernández-Mendo, A. (2009). Observación y análisis de las interacciones indirectas en el baloncesto ACB. Cuadernos de Psicología del Deporte, 9 (Suplemento), 69.

44. Wąsik, J. (2009). Structure of movement of a turning technique used in the event of special techniques in Taekwon-do ITF. Archives of Budo, 5, 111-15.

45. WTF. (2010). World Taekwondo Federation. Rules. Recuperado el 24 de Junio de 2010 de http://www.wtf.org

46. WTF. (2011). World Taekwondo Federation. History: present day. Recuperado el 15 de Mayo de 2011 de http://www.wtf.org/wtf_eng/site/ about_taekwondo/ancient_timers.html

47. Yujin, Z., Zeng, Y.J. (1999). An analysis of Chinese taekwondo team participation of men's 14th and women's 7th World Championships. Journal of Wuahu Institute of Physical Education, 33, 37-39. 